\title{
Conformal aspects of Palatini approach in Extended Theories of Gravity
}

\author{
Gianluca Allemandi, ${ }^{1, *}$ Monica Capone, ${ }^{2,}+$ Salvatore Capozziello, ${ }^{2,+}$ and Mauro Francaviglia ${ }^{1,} \S$ \\ ${ }^{1}$ Dipartimento di Matematica, Università di Torino \\ Via C. Alberto 10, 10123 TORINO (Italy) \\ ${ }^{2}$ Dipartimento di Fisica "E.R. Caianiello", Università di Salerno and INFN. Sez. di Napoli \\ Via S. Allende, I-84081 Baronissi (SA) (Italy)
}

(Dated: October 24, 2018)

\begin{abstract}
The debate on the physical relevance of conformal transformations can be faced by taking the Palatini approach into account to gravitational theories. We show that conformal transformations are not only a mathematical tool to disentangle gravitational and matter degrees of freedom (passing from the Jordan frame to the Einstein frame) but they acquire a physical meaning considering the bi-metric structure of Palatini approach which allows to distinguish between spacetime structure and geodesic structure. Examples of higher-order and non-minimally coupled theories are worked out and relevant cosmological solutions in Einstein frame and Jordan frames are discussed showing that also the interpretation of cosmological observations can drastically change depending on the adopted frame.

PACS numbers: 98.80.Jk, 04.20.-q
\end{abstract}

*Electronic address: allemandi@dm.unito.it

${ }^{\dagger}$ Electronic address: capone@sa.infn.it

${ }^{\ddagger}$ Electronic address: capozziello@sa.infn.it

${ }^{\S}$ Electronic address: francaviglia@dm.unito.it 


\section{INTRODUCTION}

Einstein's General Relativity (GR) can be considered as one of the major scientific achievements of last century. For the first time, a comprehensive theory of spacetime, gravity and matter has been formulated giving rise to a new conception of the Universe. However, in the last thirty years, several shortcomings came out in the Einstein scheme and people began to investigate whether GR is the only fundamental theory capable of explaining the gravitational interaction. Such issues come, essentially, from cosmology and quantum field theory. In the first case, the presence of the Big Bang singularity, flatness and horizon problems [1] led to the statement that Standard Cosmological Model 2], based on GR and Standard Model of particle physics, is inadequate to describe the Universe at extreme regimes. On the other hand, GR is a classical theory which does not work as a fundamental theory, when one wants to achieve a full quantum description of spacetime (and then of gravity). Due to this facts and, first of all, to the lack of a definitive quantum gravity theory, alternative theories of gravity have been pursued in order to attempt, at least, a semi-classical scheme where GR and its positive results could be recovered. One of the most fruitful approaches has been that of Extended Theories of Gravity (ETG) which have become a sort of paradigm in the study of gravitational interaction based on corrections and enlargements of the traditional Einstein scheme. The paradigm consists, essentially, in adding higher-order curvature invariants and minimally or non-minimally coupled scalar fields into dynamics which come out from the effective action of quantum gravity 7]. Other motivations to modify GR come from the issue of a whole recovering of Mach principle [3] which leads to assume a varying gravitational coupling. This principle states that the local inertial frame is determined by some average of the motion of distant astronomical objects [22], so that gravitational coupling can be scale-dependent and related to some scalar field. As a consequence, the concept of "inertia" and equivalence principle have to be revised. For example, the Brans-Dicke theory is a serious attempt to define an alternative theory to the Einstein gravity: it takes into account a variable Newton gravitational constant, whose dynamics is governed by a scalar field non-minimally coupled with geometry. In such a way, Mach's principle is better implemented [3, 23, 24].

All these approaches are not the "full quantum gravity" but are needed as working schemes toward it. In any case, they are going to furnish consistent and physically reliable results. Furthermore, every unification scheme as Superstrings, Supergravity or Grand Unified Theories, takes into account effective actions where non-minimal couplings to the geometry or higher-order terms in the curvature invariants come out. Such contributions are due to one-loop or higher-loop cor- 
rections in the high-curvature regimes near the full (not yet available) quantum gravity regime [7]. Specifically, this scheme was adopted in order to deal with the quantization on curved spacetimes and the result was that the interactions among quantum scalar fields and background geometry or the gravitational self-interactions yield corrective terms in the Einstein-Hilbert Lagrangian [19]. Moreover, it has been realized that such corrective terms are inescapable if we want to obtain the effective action of quantum gravity on scales closed to the Planck length [20]. Higher-order terms in curvature invariants (such as $R^{2}, R^{\mu \nu} R_{\mu \nu}, R^{\mu \nu \alpha \beta} R_{\mu \nu \alpha \beta}, R \square R$, or $R \square^{k} R$ ) or non-minimally coupled terms between scalar fields and geometry ( such as $\phi^{2} R$ ) have to be added to the effective Lagrangian of gravitational field when quantum corrections are considered. For instance, one can notice that such terms occur in the effective Lagrangian of strings or in Kaluza-Klein theories, when the mechanism of dimensional reduction is used [21].

From a conceptual point of view, there would be no a priori reason to restrict the gravitational Lagrangian to a linear function of the Ricci scalar $R$, minimally coupled with matter [17]. Furthermore, the idea that there are no "exact" laws of physics but that the Lagrangians of physical interactions are "stochastic" functions - with the property that local gauge invariances (i.e. conservation laws) are well approximated in the low energy limit and that physical constants can vary - has been taken into serious consideration - see Ref. [18].

Besides fundamental physics motivations, all these theories have acquired a huge interest in cosmology due to the fact that they "naturally" exhibit inflationary behaviours able to overcome the shortcomings of Standard Cosmological Model (based on GR). The related cosmological models seem very realistic and, several times, capable of matching with the observations [4, 5]. Furthermore, it is possible to show that, via conformal transformations, the higher-order and non-minimally coupled terms always correspond to Einstein gravity plus one or more than one minimally coupled scalar fields [25, 26, 27, 28]. More precisely, higher-order terms always appear as a contribution of order two in the equations of motion. For example, a term like $R^{2}$ gives fourth order equations [29], $R \square R$ gives sixth order equations [28, 30], $R \square^{2} R$ gives eighth order equations 31] and so on. By a conformal transformation, any 2nd-order of derivation corresponds to a scalar field: for example, fourth-order gravity gives Einstein plus one scalar field, sixth order gravity gives Einstein plus two scalar fields and so on [28, 32]. This feature results very interesting if we want to obtain multiple inflationary events since an early stage could select "very" large-scale structures (clusters of galaxies today), while a late stage could select "small" large-scale structures (galaxies today) [30]. The philosophy is that each inflationary era is connected with the dynamics of a scalar field. Furthermore, these extended schemes naturally could solve the problem of "graceful exit" 
bypassing the shortcomings of former inflationary models [5, 33$]$.

However, in the weak-field-limit approximation, these theories are expected to reproduce GR which, in any case, is experimentally tested only in this limit [8]. This fact is matter of debate since several relativistic theories do not reproduce exactly Einstein results in the Newtonian approximation but, in some sense, generalize them. As it was firstly noticed by Stelle [9], a $R^{2}$-theory gives rise to Yukawa-like corrections to the Newtonian potential which could have interesting physical consequences. For example, some authors claim to explain the flat rotation curves of galaxies by using such terms [10]. Others 11] have shown that a conformal theory of gravity is nothing else but a fourth-order theory containing such terms in the Newtonian limit. Besides, indications of an apparent, anomalous, long-range acceleration revealed from the data analysis of Pioneer 10/11, Galileo, and Ulysses spacecrafts could be framed in a general theoretical scheme by taking corrections to the Newtonian potential into account [12]. In general, any relativistic theory of gravitation can yield corrections to the Newton potential (see for example 13]) which, in the post-Newtonian (PPN) formalism, could furnish tests for the same theory [8]. Furthermore the newborn gravitational lensing astronomy [14] is giving rise to additional tests of gravity over small, large, and very large scales which very soon will provide direct measurements for the variation of Newton coupling $G_{N}$ [15], the potential of galaxies, clusters of galaxies [16] and several other features of gravitating systems. Such data will be, very likely, capable of confirming or ruling out the physical consistency of GR or of any ETG.

In summary, the general feature of ETGs is that the Einstein field equations result to be modified in two senses: $i$ ) geometry can be non-minimally coupled to some scalar field, and/or ii) higher than second order derivative terms in the metric come out. In the first case, we generically deal with scalar-tensor theories of gravity or non-minimally coupled theories; in the second one we deal with higher-order theories. However several combinations of both situations can result physically interesting [27]. In this case, we deal with higher-order-scalar-tensor theories of gravity.

In all these approaches, the problem of reducing more general theories to Einstein standard form has been extensively treated; one can see that, through a "Legendre" transformation on the metric, higher-order theories, under suitable regularity conditions on the Lagrangian, take the form of the Einstein one in which a scalar field (or more than one) is the source of the gravitational field (see for example [34] 17, 35, 36]); on the other side, it has been studied the equivalence between models with variable gravitational coupling with the Einstein standard gravity through a suitable conformal transformation (see [38, 39]). In any case, the debate on the physical meaning of conformal transformations is far to be solved [see [40] and refererences therein for a comprehensive 
review]. Several authors claim for a true physical difference between Jordan frame (higher-order theories and/or variable gravitational coupling) since there are experimental and observational evidences which point out that the Jordan frame could be suitable to better match solutions with data. Others state that the true physical frame is the Einstein one according to the energy theorems 36]. In any case, the discussion is open and no definite statement has been done up to now. The problem should be faced from a more general viewpoint and the Palatini approach to gravity could be useful to this goal. The Palatini approach in gravitational theories was firstly introduced and analyzed by Einstein himself 45 . It was however called Palatini approach as a consequence of an historical misunderstanding [46, 47]. The fundamental idea at the bases of the Palatini formalism is to consider the (usually torsion-less) connection $\Gamma$, entering the definition of the Ricci tensor to be independent of the metric $g$ defined on the spacetime $M$. The Palatini formalisms for the standard Hilbert-Einstein torsion-less theory results to be equivalent to the purely metric theory: this follows from the fact that the field equation for the connection fields states exactly that the same connection $\Gamma$, firstly considered to be independent, should be the Levi-Civita connection of the metric $g$. There is consequently no reason to impose the Palatini variational principle in the standard Hilbert-Einstein theory instead of the metric (Einstein) variational principle. The situation however completely changes when we consider the case of ETGs, depending on analytical functions of curvature invariants as $f(R)$, or non-minimally coupled a scalar field. In these cases, as we will show later in detail, the Palatini and the metric variational principle provide different field equations and the theories thus derived surely differ; see for a partial discussion [48] and [36]. The importance of the Palatini approach in this framework has been recently proven in relation with cosmological applications [49, 50].

From a physical viewpoint, considering the metric $g$ and the connection $\Gamma$ as independent fields is somehow equivalent to decouple the metric structure of spacetime and its geodesic structure (i.e. the connection is not the Levi-Civita connection of $g$ ), governing respectively the chronological structure of spacetime and the trajectories of particles, moving in it. This decoupling enriches the geometrical structure of spacetime and generalizes the purely metric formalism. This metric-affine structure of spacetime (here, we simply mean that a connection $\Gamma$ and a metric $g$ are involved) is naturally translated, by means of the same (Palatini) field equations, into a bi-metric structure of spacetime. Besides the physical metric $g$, another metric $h$ is involved. This new metric, at least in $f(R)$ theories, is simply related to the connection. As a matter of facts, the connection $\Gamma$ results to be the Levi-Civita connection of $h$ and thus provides the geodesic structure of spacetime. When we consider the case of non-minimally coupled interaction in the gravitational Lagrangian 
(scalar-tensor theories), the new metric $h$ is somehow related with the non-minimal coupling. Also in the case of Brans-Dicke like theories the new metric $h$ can be thus related to a different geometrical and physical aspect of the gravitational theory. Thanks to the Palatini formalism this non-minimal coupling effects and the scalar field, entering the evolution of the gravitational fields, are separated from the metric structure of spacetime. The situation mixes when we consider the case of higher-order-scalar-tensor theories.

In this paper we analyze, through appropriately defined conformal transformations, the problem of the equivalence between higher-order non-minimally coupled theories and General Relativity in the Palatini approach. First, we will do it in the general context of the field theories and then we reduce to the cosmological case, that is, we will study the conformal invariance under the hypotheses of homogeneity and isotropy. In this case, we also consider the case in which ordinary matter is present, besides the scalar field, and we make some consideration on the problem of which is the "physical system" between the two conformally equivalent systems [3, 17, 35, 36].

The layout of the paper is the following. In Sec.II, we discuss the conformal transformations through their applications to non-minimally coupled and higher-order theories of gravity. The goal is to show that starting from the Jordan frame (at least in the case in which standard perfect fluid matter is not considered), through a conformal transformation, the system can always be reduced to the Einstein frame, where gravity is minimally coupled to one (or more than one) scalar field(s). In principle, every ETG is conformally equivalent to GR+scalar field(s). Sec.III is devoted to the discussion of conformal transformations in the framework of Palatini approach. Due to the intrinsic bi-metric structure of such an approach and to the fact that affine connections coincide with LeviCivita connection only in the Hilbert-Einstein case, conformal transformations acquire a relevant role in order to study chronological and geodesic structures of spacetime. In other words, they are not only a mere mathematical tool but they put in evidence that physics could be different in Einstein and Jordan frame. This last issue is particularly highlighted in cosmology, as discussed in Sec.IV. In fact, the solutions derived in the different frames could be distinguished by observations. This fact shows that Einstein frame and Jordan frame are physically inequivalent since Palatini field equations are intrinsically different from those derived in standard GR. Conclusions are drawn in Sec.V. 


\section{CONFORMAL TRANSFORMATIONS}

Let us start giving detailed examples of conformal transformations in order to show how they work on the Lagrangian and the field equations of a given ETG, defined in the Jordan frame. The goal is to reduce the theory to the Einstein frame, i.e. to a minimally coupled theory plus decoupled scalar field(s). The procedure, in principle, works for any ETG, but it is extremely useful, as we will see below, in the interpretation of solutions which, from a physical viewpoint, should be fitted against experimental and observational data. Before discussing conformal transformations in the framework of the Palatini approach, we work out in detail the purely metric non-minimally coupled scalar-tensor case and the $f(R)$ case, giving general considerations at the end of this section.

\section{A. The Scalar-Tensor case}

In four dimensions, a general non-minimally coupled scalar-tensor theory of gravity is given by the effective (purely metric) action

$$
\mathcal{A}=\int d^{4} x \sqrt{-g}\left[F(\phi) R+\frac{1}{2} g^{\mu \nu} \phi_{; \mu} \phi_{; \nu}-V(\phi)\right]
$$

where $R$ is the Ricci scalar, $V(\phi)$ and $F(\phi)$ are generic functions describing respectively the potential and the coupling of $\phi$. We shall adopt Planck units. The Brans-Dicke theory of gravity is a particular case of the action (11) for $V(\phi)=0$ [37]. The variation with respect to $g_{\mu \nu}$ gives the field equations

$$
F(\phi) G_{\mu \nu}=F(\phi)\left[R_{\mu \nu}-\frac{1}{2} R g_{\mu \nu}\right]=-\frac{1}{2} T_{\mu \nu}-g_{\mu \nu} \square_{g} F(\phi)+F(\phi)_{; \mu \nu}
$$

which are the generalized Einstein equations; here $\square_{\Gamma}$ is the d'Alembert operator with respect to the metric $g$, and $G_{\mu \nu}$ is the Einstein tensor. Here and below, semicolon denotes metric covariant derivatives with respect to $g$. The energy-momentum tensor relative to the scalar field is

$$
T_{\mu \nu}=\phi_{; \mu} \phi_{; \nu}-\frac{1}{2} g_{\mu \nu} \phi_{; \alpha} \phi_{;}^{\alpha}+g_{\mu \nu} V(\phi)
$$

The variation with respect to $\phi$ provides the Klein-Gordon equation

$$
\square_{g} \phi-R F_{\phi}(\phi)+V_{\phi}(\phi)=0
$$

where $F_{\phi}=d F(\phi) / d \phi, V_{\phi}=d V(\phi) / d \phi$. This last equation is equivalent to the Bianchi contracted identity [52]. The conformal transformation on the metric $g_{\mu \nu}$ is

$$
\bar{g}_{\mu \nu}=e^{2 \omega} g_{\mu \nu}
$$


in which $e^{2 \omega}$ is the conformal factor. Under this transformation, the Lagrangian density in (11) becomes

$$
\begin{aligned}
\sqrt{-g}\left(F R+\frac{1}{2} g^{\mu \nu} \phi_{; \mu} \phi_{; \nu}-V\right) & =\sqrt{-\bar{g}} e^{-2 \omega}\left(F \bar{R}-6 F \square_{\bar{g}} \omega+\right. \\
& \left.-6 F \omega_{; \alpha} \omega_{;}^{\alpha}+\frac{1}{2} \bar{g}^{\mu \nu} \phi_{; \mu} \phi_{; \nu}-e^{-2 \omega} V\right)
\end{aligned}
$$

in which $\bar{R}$ and $\square_{\bar{g}}$ are respectively the Ricci scalar and the d'Alembert operator relative to the metric $\bar{g}$. Requiring the theory in the metric $\bar{g}_{\mu \nu}$ to appear as a standard Einstein theory, the conformal factor has to be related to $F$ [39], that is

$$
e^{2 \omega}=-2 F
$$

F must be negative to restore physical coupling. Using this relation, the Lagrangian density (6) becomes

$$
\sqrt{-g}\left(F R+\frac{1}{2} g^{\mu \nu} \phi_{; \mu} \phi_{; \nu}-V\right)=\sqrt{-\bar{g}}\left(-\frac{1}{2} \bar{R}+3 \square_{\bar{g}} \omega+\frac{3 F_{\phi}^{2}-F}{4 F^{2}} \phi_{; \alpha} \phi_{;}^{\alpha}-\frac{V}{4 F^{2}}\right),
$$

Introducing a new scalar field $\bar{\phi}$ and the potential $\bar{V}$, respectively, defined by

$$
\bar{\phi}_{; \alpha}=\sqrt{\frac{3 F_{\phi}^{2}-F}{2 F^{2}}} \phi_{; \alpha}, \quad \bar{V}(\bar{\phi}(\phi))=\frac{V(\phi)}{4 F^{2}(\phi)},
$$

we get

$$
\sqrt{-g}\left(F R+\frac{1}{2} g^{\mu \nu} \phi_{; \mu} \phi_{; \nu}-V\right)=\sqrt{-\bar{g}}\left(-\frac{1}{2} \bar{R}+\frac{1}{2} \bar{\phi}_{; \alpha} \bar{\phi}_{;}^{\alpha}-\bar{V}\right)
$$

which is the usual Hilbert-Einstein Lagrangian density plus the standard Lagrangian density relative to the scalar field $\bar{\phi}$. (We have not considered the divergence-type term appearing in the Lagrangian (10); we will return on this point in our forthcoming considerations). Therefore, every non-minimally coupled scalar-tensor theory, in absence of ordinary matter, i.e. perfect fluid, is conformally equivalent to an Einstein theory, being the conformal transformation and the potential suitably defined by (7) and (9). The converse is also true: for a given $F(\phi)$, such that $3 F_{\phi}^{2}-F>0$, we can transform a standard Einstein theory into a non-minimally coupled scalar-tensor theory. This means that, in principle, if we are able to solve the field equations in the framework of the Einstein theory in presence of a scalar field with a given potential, we should be able to get the solutions for the scalar-tensor theories, assigned by the coupling $F(\phi)$, via the conformal transformation (77) with the constraints given by Eqs.(91). This is exactly what we are going to discuss in the cosmological context in cases in which the potentials as well as the couplings are relevant 
from the point of view of the fundamental physics. In our opinion, this is not only a mathematical procedure but, by the Palatini approach, it is related to the physical degrees of freedom of the theory which are, in some sense, "disentangled" by the conformal transformations.

Following the standard terminology, the "Einstein frame" is the framework of the Einstein theory with the minimal coupling and the "Jordan frame" is the framework of the non-minimally coupled theory [44].

We have to make some interesting remarks with respect to (8) and (9): the "new" scalar field, defined in (92) is given in differential form in terms of the "old" one and its integration can be not trivial; the second remark concerns the divergence appearing in (8). The transformed Lagrangian density obtained from (6) by imposing (77) contains a divergence term, in which not only the metric but also its derivative appear, through the connection $\bar{\Gamma}$. Therefore the equivalence of this total Lagrangian density to the Hilbert-Einstein Lagrangian density plus scalar field is not trivial and, due to this fact, the Palatini approach, which distinguishes a priori the fields $g$ and $\Gamma$, is extremely relevant. To check that they are actually equivalent, let us perform the conformal transformation (5) on the field Eqs. (2), obtaining

$$
\begin{aligned}
\bar{G}_{\mu \nu} & =\left(-\frac{1}{2 F}+\frac{F_{\phi \phi}}{F}+\frac{2 \omega_{\phi} F_{\phi}}{F}-2 \omega_{\phi}^{2}-2 \omega_{\phi \phi}\right) \phi_{; \mu} \phi_{; \nu}+ \\
& +\left(\frac{1}{4 F}-\frac{F_{\phi \phi}}{F}+\frac{\omega_{\phi} F_{\phi}}{F}-\omega_{\phi}^{2}+2 \omega_{\phi \phi}\right) \bar{g}_{\mu \nu} \phi_{; \alpha} \phi_{;}^{\alpha}+\left(-\frac{F_{\phi}}{F}+2 \omega_{\phi}\right) \bar{g}_{\mu \nu} \square_{\bar{\Gamma}} \phi+ \\
& +\left(\frac{F_{\phi}}{F}-2 \omega_{\phi}\right)\left(\nabla^{\bar{\Gamma}}\right)_{\mu}\left(\nabla^{\bar{\Gamma}}\right)_{\nu} \phi-\frac{1}{2 F} e^{-2 \omega_{\bar{g}}}{ }_{\mu \nu} V
\end{aligned}
$$

in which $\left(\nabla^{\bar{\Gamma}}\right)_{\mu}$ is the covariant derivative with respect to $x^{\mu}$ relative to the connection $\bar{\Gamma}$ and $\square_{\bar{\Gamma}}$ is nothing but $\square_{\bar{g}}$. If $\omega$ satisfies the relation

$$
\frac{F_{\phi}}{F}-2 \omega_{\phi}=0
$$

Eqs. (11) can be rewritten as

$$
\bar{G}_{\mu \nu}=\frac{3 F_{\phi}^{2}-F}{2 F^{2}} \phi_{; \mu} \phi_{; \nu}-\bar{g}_{\mu \nu} \frac{3 F_{\phi}^{2}-F}{2 F^{2}} \phi_{; \alpha} \phi_{;}^{\alpha}-\bar{g}_{\mu \nu} \frac{e^{-2 \omega}}{2 F} V .
$$

Then, using the transformations (9) and defining the potential

$$
W(\bar{\phi}(\phi))=-\frac{e^{-2 \omega(F)}}{2 F} V,
$$

where $\omega(F)$ satisfies (12), Eq. (13) becomes

$$
\bar{G}_{\mu \nu}=\bar{\phi}_{; \mu} \bar{\phi}_{; \nu}-\frac{1}{2} \bar{g}_{\mu \nu} \bar{\phi}_{; \alpha} \bar{\phi}_{;}^{\alpha}-\bar{g}_{\mu \nu} W,
$$


which correspond to the Einstein field equations in presence of a scalar field $\bar{\phi}$ with potential $W$. The function $\omega(F)$ is obtained from (12), that is

$$
\omega=\frac{1}{2} \ln F+\omega_{0}
$$

in which $\omega_{0}$ is an integration constant. The potential $W$ takes the form

$$
W=-\frac{V}{2 \xi F}
$$

From (17) and the second of (9), we see that, fixing $\xi=-2$, the definition of $W$ coincides with that one of $\bar{V}$. We have then the full compatibility with the Lagrangian approach obtaining for $\omega$ the relation (7); in this sense, the equivalence between the non-minimally coupled Lagrangian density and the Hilbert-Einstein Lagrangian density plus scalar field is verified.

A final remark regards Eqs.(9): actually, from (8) the relation between $\bar{\phi}_{; \alpha}$ and $\phi_{; \alpha}$ present $\mathrm{a} \pm$ sign in front of the square root, which corresponds to have the same or opposite sign in the derivative of $\phi$ and $\bar{\phi}$ with respect to $x_{\alpha}$. This ambiguity acquire a physical meaning in the interpretation of the scalar field, as we shall see below.

\section{B. Higher-order gravity case}

In general, fourth-order theories of gravity are given by the action

$$
\mathcal{A}=\int d^{4} x \sqrt{-g} f(R)
$$

where $f(R)$ is an analytic function of the Ricci curvature scalar $R$. We are considering the simplest case of fourth-order gravity but we can construct such kind of theories also using the invariants $R_{u \nu}$ о $R_{\gamma \mu \nu}^{\alpha}$. However, for cosmological considerations, theories like (18) are sufficiently general 54]. Hilbert-Einstein action is recovered for $f(R)=R$. Varying with respect to $g_{\alpha \beta}$, we get the field equations

$$
f^{\prime}(R) R_{\alpha \beta}-\frac{1}{2} f(R) g_{\alpha \beta}=f^{\prime}(R)^{;^{\mu \nu}}\left(g_{\alpha \mu} g_{\beta \nu}-g_{\alpha \beta} g_{\mu \nu}\right)
$$

which are fourth-order equations thanks to the term $f^{\prime}(R)^{; \mu \nu}$. The prime indicates the derivative with respect to $R$. Putting in evidence the Einstein tensor, we have

$$
G_{\alpha \beta}=\frac{1}{f^{\prime}(R)}\left\{\frac{1}{2} g_{\alpha \beta}\left[f(R)-R f^{\prime}(R)\right]+f^{\prime}(R)_{; \alpha \beta}-g_{\alpha \beta} \square f^{\prime}(R)\right\},
$$


where the gravitational contributions in the stress-energy tensor can be interpreted, via conformal transformations, as scalar field contributions and then as "matter" terms. Performing the conformal transformation (5), we get

$$
\begin{aligned}
& \bar{G}_{\alpha \beta}=\frac{1}{f^{\prime}(R)}\left\{\frac{1}{2} g_{\alpha \beta}\left[f(R)-R f^{\prime}(R)\right]+f^{\prime}(R)_{; \alpha \beta}-g_{\alpha \beta} \square f^{\prime}(R)\right\}+ \\
&+2\left(\omega_{; \alpha ; \beta}+g_{\alpha \beta} \square \omega-\omega_{; \alpha \beta}+\frac{1}{2} g_{\alpha \beta} \omega_{; \gamma} \omega^{; \gamma}\right) .
\end{aligned}
$$

We can choose the conformal factor

$$
\omega=\frac{1}{2} \ln \left|f^{\prime}(R)\right|
$$

which has to be substituted into (21). Rescaling $\omega$ in such a way that

$$
k \phi=\omega,
$$

and $k=\sqrt{1 / 6}$, we obtain the Lagrangian equivalence

$$
\sqrt{-g} f(R)=\sqrt{-\bar{g}}\left(-\frac{1}{2} \bar{R}+\frac{1}{2} \bar{\phi}_{; \alpha} \bar{\phi}_{;}^{\alpha}-\bar{V}\right)
$$

and the Einstein equations in standard form

$$
\bar{G}_{\alpha \beta}=\phi_{; \alpha} \phi_{; \beta}-\frac{1}{2} \bar{g}_{\alpha \beta} \phi_{; \gamma} \phi^{; \gamma}+\bar{g}_{\alpha \beta} V(\phi)
$$

with the potential

$$
V(\phi)=\frac{e^{-4 k \phi}}{2}\left[f(\phi)-\mathcal{F}\left(e^{2 k \phi}\right) e^{2 k \phi}\right]=\frac{1}{2} \frac{f(R)-R f^{\prime}(R)}{f^{\prime}(R)^{2}} .
$$

$\mathcal{F}$ is the inverse function of $f^{\prime}(\phi)$ and $f(\phi)=\int \exp (2 k \phi) d \mathcal{F}$. However, the problem is completely solved if $f^{\prime}(\phi)$ can be analytically inverted. In summary, a fourth-order theory is conformally equivalent to the standard second-order Einstein theory plus a scalar field (see also [17, 34|).

If the theory is higher than fourth order, we have Lagrangian densities of the form [28, 30, 47],

$$
\mathcal{L}=\mathcal{L}\left(R, \square R, \ldots \square^{k} R\right)
$$

Every $\square$ operator introduces two further terms of derivation into the field equations. For example a theory like

$$
\mathcal{L}=R \square R,
$$


is a sixth-order theory, and the above approach can be pursued considering a conformal factor of the form

$$
\omega=\frac{1}{2} \ln \left|\frac{\partial \mathcal{L}}{\partial R}+\square \frac{\partial \mathcal{L}}{\partial \square R}\right|
$$

In general, increasing two orders of derivation in the field equations (i.e. every term $\square R$ ), corresponds to add a scalar field in the conformally transformed frame [28]. A sixth-order theory can be reduced to an Einstein theory with two minimally coupled scalar fields; a $2 n$-order theory can be, in principle, reduced to an Einstein theory $+(n-1)$-scalar fields. On the other hand, these considerations can be directly generalized to higher-order-scalar-tensor theories in any number of dimensions as shown in [26].

As concluding remarks, we can say that conformal transformations works at three levels: $i$ ) on the Lagrangian of the given ETG-theory; ii) on the field equations; iii) on the solutions. The table below summarizes the situation for fourth-order gravity (FOG), non-minimally coupled scalartensor theories (NMC) and standard Hilbert-Einstein (HE) theory. Clearly, direct and inverse transformations correlate all the steps of the table but no absolute criterion, at this point of the discussion, is capable of stating what is the "physical" framework since from a mathematical point of view all the frames are equivalent (see also [36] for a detailed discussion). The Palatini approach can aid in this task.

\begin{tabular}{|ccccc|}
\hline $\mathcal{L}_{\text {FOG }}$ & $\longleftrightarrow$ & $\mathcal{L}_{N M C}$ & $\longleftrightarrow$ & $\mathcal{L}_{H E}$ \\
$\uparrow$ & & $\uparrow$ & & $\uparrow$ \\
FOG Eqs. & & NMC Eqs. & $\longleftrightarrow$ & Einstein Eqs. \\
$\uparrow$ & & $\uparrow$ & & $\uparrow$ \\
FOG Solutions & $\longleftrightarrow$ & NMC Solutions & $\longleftrightarrow$ & Einstein Solutions \\
\hline
\end{tabular}

\section{PALATINI APPROACH AND CONFORMAL TRANSFORMATIONS}

As we said, the Palatini approach, considering $g$ and $\Gamma$ as independent fields, is "intrinsically" bimetric and capable of disentangle the geodesic from the chronological structure of a given manifold. Starting from these features for ETG, conformal transformations assume a fundamental role in defining the affine connection which is merely "Levi-Civita" only for Hilbert-Einstein gravity. In this section, we work out examples showing how conformal transformations assume a fundamental physical role. 


\section{A. $\quad f(R)$ gravity in Palatini approach and the intrinsic conformal structure}

Let us start from the case of fourth-order gravity where Palatini variational principle is straightforward in showing the differences with Hilbert-Einstein variational principle, involving only metric. Besides cosmological applications of $f(R)$ gravity have shown the importance of the Palatini formalism in this framework, giving physically relevant results and avoiding singular behaviors of solutions [49, 50]. This last nice feature is not present in the standard metric approach. The standard and more general $f(R)$ Lagrangian suitable for our considerations is

$$
A=A_{\text {grav }}+A_{\text {mat }}=\int \sqrt{-g}\left[f(R)+2 \kappa L_{\text {mat }}(\Psi)\right] d^{4} x
$$

where $R \equiv R(g, \Gamma)=g^{\alpha \beta} R_{\alpha \beta}(\Gamma)$ is the generalized Ricci scalar and $R_{\mu \nu}(\Gamma)$ is the Ricci tensor of a torsionless connection $\Gamma$, which a priori has no relations with the metric $g$ of spacetime. The gravitational part of the Lagrangian is controlled by a given real analytical function of one real variable $f(R)$, while $\sqrt{-g}$ denotes a related scalar density of weight 1 . The Lagrangian contains also a matter part, usually chosen to be the Lagrangian of the perfect fluid $L_{\text {mat }}$ in minimal interaction with the gravitational field but it can be also a minimally coupled scalar field(s) Lagrangian. This Lagrangian is dependent on matter fields $\Psi$ together with their first derivatives and equipped with a gravitational coupling constant $\kappa=8 \pi G$ which we restore now, with respect to the previous considerations for the sake of clearness in the discussion. More general couplings between the gravitational Lagrangian and matter fields, involving the covariant derivatives with respect to $\Gamma$ of matter fields could be considered. We remark, however, that the absence of these interactions do not change much the physics of the theory, owing to the conformal relation in the bi-metric structure of spacetime in $f(R)$ gravity; see [49] for details. Field equations, deriving from the Palatini variational principle are (we assume the spacetime manifold to be a Lorentzian manifold $\mathcal{M}$ with $\operatorname{dim} \mathcal{M}=4 ;$ see [48]):

$$
\begin{gathered}
f^{\prime}(R) R_{(\mu \nu)}(\Gamma)-\frac{1}{2} f(R) g_{\mu \nu}=\kappa T_{\mu \nu} \\
\nabla_{\alpha}^{\Gamma}\left(\sqrt{-g} f^{\prime}(R) g^{\mu \nu}\right)=0
\end{gathered}
$$

where $T_{\mu \nu}=-2 \frac{\delta L_{\mathrm{mat}}}{\delta g_{\mu \nu}}$ denotes the matter source stress-energy tensor and $\nabla^{\Gamma}$ is the covariant derivative with respect to $\Gamma$. We shall use the standard notation denoting by $R_{(\mu \nu)}$ the symmetric part of $R_{\mu \nu}$, i.e. $R_{(\mu \nu)} \equiv \frac{1}{2}\left(R_{\mu \nu}+R_{\nu \mu}\right)$. In order to get (32) one has to additionally assume that $L_{\text {mat }}$ is functionally independent of $\Gamma$ (as already remarked); however it may contain metric 
covariant derivatives $\stackrel{g}{\nabla}$ of fields. This means that the matter stress-energy tensor $T_{\mu \nu}=T_{\mu \nu}(g, \Psi)$ depends on the metric $g$ and some matter fields denoted here by $\Psi$, together with their derivatives (covariant derivatives with respect to the Levi-Civita connection of $g$ ). From (32) one sees that $\sqrt{-g} f^{\prime}(R) g^{\mu \nu}$ is a symmetric twice contravariant tensor density of weight 1 . As previously discussed in [49] and [48] this naturally lead us to define a new metric $h_{\mu \nu}$, such that the following relation holds true:

$$
\sqrt{-g} f^{\prime}(R) g^{\mu \nu}=\sqrt{-h} h^{\mu \nu}
$$

This ansatz is suitably made in order to impose $\Gamma$ to be the Levi-Civita connection of $h$ and the only restriction is that $\sqrt{-g} f^{\prime}(R) g^{\mu \nu}$ should be non-degenerate. In the case of Hilbert-Einstein Lagrangian, $f^{\prime}(R)=1$ and the statement is trivial. The above equation (33) imposes that the two metrics $h$ and $g$ are conformally equivalent. The corresponding conformal factor can be easily found to be $f^{\prime}(R)$ (in $\operatorname{dim} \mathcal{M}=4$ ) and the conformal transformation results to be ruled by:

$$
h_{\mu \nu}=f^{\prime}(R) g_{\mu \nu}
$$

Therefore, as it is well known, equation (32) implies that $\Gamma=\Gamma_{L C}(h)$ and $R_{(\mu \nu)}(\Gamma)=R_{\mu \nu}(h) \equiv$ $R_{\mu \nu}$. Field equations can be supplemented by the scalar-valued equation obtained by taking the trace of (31), (we define $\tau=\operatorname{tr} \hat{T}$ )

$$
f^{\prime}(R) R-2 f(R)=\kappa g^{\alpha \beta} T_{\alpha \beta} \equiv \kappa \tau
$$

which controls solutions of (32). We shall refer to this scalar-valued equation as the structural equation of spacetime. In the vacuum case (and radiating spacetimes, such that $\tau=0$ ) this scalarvalued equation admits only constant solutions and the universality of Einstein field equations holds [48], corresponding to a theory with constant cosmological constant [51]. In the case of interaction with matter fields, the structural equation (34), if explicitly solvable, provides an expression of $R=F(\tau)$ and consequently both $f(R)$ and $f^{\prime}(R)$ can be expressed in terms of $\tau$. The matter content of spacetime thus rules the bi-metric structure of spacetime and consequently both the geodesic and metric structures [49] which are intrinsically different. This behavior generalizes the vacuum case and corresponds to the case of a time varying cosmological constant. In other words, due to these features, conformal transformations, which allow to pass from a metric structure to another one, acquire an intrinsic physical meaning since "select" metric and geodesic structures which for a given ETG, in principle, do not coincide. 


\section{B. The case of Scalar-Tensor Gravity}

Let us now try to extend the above formalism to case of non-minimally coupled scalar-tensor theories. The effort is to understand if and how the bi-metric structure of spacetime behaves in this cases and which could be its geometrical and physical interpretation. As a general result, the Palatini formalism and the bi-metric structure "select" intrinsically different theories of gravity. The importance of these theories is well known in cosmological applications. For completeness reasons we start by considering scalar-tensor theories in the Palatini formalism, calling $A_{1}$ the action functional. After, we take into account the case of decoupled non-minimal interaction between a scalar-tensor theory and a $f(R)$ theory, calling $A_{2}$ this action functional. We finally consider the case of non-minimal-coupled interaction between the scalar field $\phi$ and the gravitational fields $(g, \Gamma)$, calling $A_{3}$ the corresponding action functional. Particularly significant is, in this case, the limit of low curvature $R$. This resembles the physical relevant case of present values of curvatures of the universe and it is important for cosmological applications.

The action (1) for scalar-tensor gravity can be generalized, in order to better develop the Palatini approach, as:

$$
A_{1}=\int \sqrt{-g}\left[F(\phi) R+\frac{\varepsilon}{2} \stackrel{g}{\nabla}_{\mu} \phi \stackrel{g}{\nabla}^{\mu} \phi-V(\phi)+\kappa L_{\mathrm{mat}}(\Psi, \stackrel{g}{\nabla} \Psi)\right] d^{4} x
$$

where $\phi$, as above, is an arbitrary scalar field. The values of $\varepsilon= \pm 1$ selects between standard scalar field theories and quintessence field theories. The relative "signature" can be selected by conformal transformations. Field equations for the gravitational part of the action are, respectively for the metric $g$ and the connection $\Gamma$ :

$$
\left\{\begin{array}{l}
F(\phi)\left[R_{(\mu \nu)}-\frac{1}{2} R g_{\mu \nu}\right]=\kappa\left[T_{\mu \nu}^{\phi}+T_{\mu \nu}^{\mathrm{mat}}\right] \\
\nabla_{\alpha}^{\Gamma}\left(\sqrt{-g} F(\phi) g^{\mu \nu}\right)=0
\end{array}\right.
$$

where we have defined the stress-energy tensors for the scalar field and the matter Lagrangian, respectively as:

$$
\left\{\begin{array}{l}
T_{\mu \nu}^{\phi}=-\frac{2}{\kappa} \frac{\delta L_{\phi}}{\delta g_{\mu \nu}} \\
T_{\mu \nu}^{\mathrm{mat}}=-2 \frac{\delta L_{\mathrm{mat}}}{\delta g_{\mu \nu}}
\end{array}\right.
$$

and $R_{(\mu \nu)}$ is the same defined in (31). For matter fields we have the following field equations:

$$
\left\{\begin{array}{l}
\varepsilon \square \phi=-V_{\phi}(\phi)+F_{\phi}(\phi) R \\
\frac{\delta L_{\mathrm{mat}}}{\delta \Psi}=0
\end{array}\right.
$$

In this case, the structural equation of spacetime implies that:

$$
R=-\frac{\kappa\left(\tau^{\phi}+\tau^{\mathrm{mat}}\right)}{F(\phi)}
$$


which expresses the value of the Ricci scalar curvature in terms of traces of the stress-energy tensors of matter (we have to require $F(\phi) \neq 0$ ). The bi-metric structure of spacetime is thus defined by the ansatz:

$$
\sqrt{-g} F(\phi) g^{\mu \nu}=\sqrt{-h} h^{\mu \nu}
$$

such that $g$ and $h$ result to be conformal:

$$
h_{\mu \nu}=F(\phi) g_{\mu \nu}
$$

The conformal factor is exactly the minimal interaction factor. We remark that from (40) it follows that in the vacuum case $\tau^{\phi}=0$ and $\tau^{\text {mat }}=0$ this theory is equivalent to the standard Einstein one without matter. On the other hand, for $F(\phi)=F_{0}$ we recover the Einstein theory plus a minimally coupled scalar field. As last remark and keeping in mind the discussion of the previous section, the Palatini approach intrinsically furnishes the conformal structure (42) of the theory which is trivial in the Einstein, minimally coupled case.

\section{Decoupled non-minimal interaction in scalar-tensor $f(R)$ theories}

As a further step, we generalize the results of previous subsection, considering the case of a non-minimal coupling in the framework of $f(R)$ theories. The action functional can be written as:

$$
A_{2}=\int \sqrt{-g}\left[F(\phi) f(R)+\frac{\varepsilon}{2} \stackrel{g}{\nabla}_{\mu} \phi \stackrel{g}{\nabla}^{\mu} \phi-V(\phi)+\kappa L_{\text {mat }}(\Psi, \stackrel{g}{\nabla} \Psi)\right] d^{4} x
$$

where $f(R)$ is, as usual, any analytical function of the Ricci scalar $R$. Field equations (in the Palatini formalism) for the gravitational part of the action are:

$$
\left\{\begin{array}{l}
F(\phi)\left[f^{\prime}(R) R_{(\mu \nu)}-\frac{1}{2} f(R) g_{\mu \nu}\right]=\kappa\left[T_{\mu \nu}^{\phi}+T_{\mu \nu}^{\mathrm{mat}}\right] \\
\nabla_{\alpha}^{\Gamma}\left(\sqrt{-g} F(\phi) f^{\prime}(R) g^{\mu \nu}\right)=0
\end{array}\right.
$$

where we have defined the stress-energy tensors for the scalar field and the matter Lagrangian, respectively as in Eqs.(38). For scalar and matter fields we have otherwise the following field equations:

$$
\left\{\begin{array}{l}
\varepsilon \square \phi=-V_{\phi}(\phi)+\sqrt{-g} F_{\phi}(\phi) f(R) \\
\frac{\delta L_{\text {mat }}}{\delta \Psi}=0
\end{array}\right.
$$

where the non-minimal interaction term enters into the modified Klein-Gordon equations. In this case the structural equation of spacetime implies that:

$$
f^{\prime}(R) R-2 f(R)=\frac{\kappa\left(\tau^{\phi}+\tau^{\mathrm{mat}}\right)}{F(\phi)}
$$


We remark that this equation, if solved, expresses the value of the Ricci scalar curvature in terms of traces of the stress-energy tensors of matter (we have to require again that $F(\phi) \neq 0$ ). The bi-metric structure of spacetime is thus defined by the ansatz:

$$
\sqrt{-g} F(\phi) f^{\prime}(R) g^{\mu \nu}=\sqrt{-h} h^{\mu \nu}
$$

such that $g$ and $h$ result to be conformally related by:

$$
h_{\mu \nu}=F(\phi) f^{\prime}(R) g_{\mu \nu}
$$

We remark that, once the structural equation is solved, the conformal factor depends on the values of the matter fields $(\phi, \Psi)$ or, more precisely, on the traces of the stress-energy tensors and the value of $\phi$. From equation (46), it follows that in the case of vacuum, i.e. in the case that both $\tau^{\phi}=0$ and $\tau^{\text {mat }}=0$, the universality of Einstein field equation still holds as in the case of minimally interacting $f(R)$ theories [48]. The proof is very simply, as it follows exactly the headlines of [48]. The validity of this property is related to the decoupling of the scalar field and the gravitational field in this case.

\section{The general case}

Let us finally consider the case where the gravitational Lagrangian is a general function of $\phi$ and $R$. The action functional can thus be written as:

$$
A_{3}=\int \sqrt{-g}\left[K(\phi, R)+\frac{\varepsilon}{2} \stackrel{g}{\nabla}_{\mu} \phi \stackrel{g}{\nabla}^{\mu} \phi-V(\phi)+\kappa L_{\mathrm{mat}}(\Psi, \stackrel{g}{\nabla} \Psi)\right] d^{4} x
$$

Field equations for the gravitational part of the action are:

$$
\left\{\begin{array}{l}
{\left[\frac{\partial K(\phi, R)}{\partial R}\right] R_{(\mu \nu)}-\frac{1}{2} K(\phi, R) g_{\mu \nu}=\kappa\left[T_{\mu \nu}^{\phi}+T_{\mu \nu}^{\text {mat }}\right]} \\
\nabla_{\alpha}^{\Gamma}\left(\sqrt{-g}\left[\frac{\partial K(\phi, R)}{\partial R}\right] g^{\mu \nu}\right)=0
\end{array}\right.
$$

where we have defined the stress-energy tensors for the scalar field and the matter Lagrangian, respectively as in Eqs.(38). For matter fields we have the following field equations:

$$
\left\{\begin{array}{l}
\varepsilon \square \phi=-V_{\phi}(\phi)+\left[\frac{\partial K(\phi, R)}{\partial \phi}\right] \\
\frac{\delta L_{\mathrm{mat}}}{\delta \Psi}=0
\end{array}\right.
$$

The structural equation of spacetime can be expressed as:

$$
\frac{\partial K(\phi, R)}{\partial R} R-2 K(\phi, R)=\kappa\left(\tau^{\phi}+\tau^{\mathrm{mat}}\right)
$$


We remark that this equation, if solved, expresses again the form of the Ricci scalar curvature in terms of traces of the stress-energy tensors of matter (we should impose regularity conditions and, for example, $K(\phi, R) \neq 0)$. The bi-metric structure of spacetime is thus defined by the ansatz:

$$
\sqrt{-g} \frac{\partial K(\phi, R)}{\partial R} g^{\mu \nu}=\sqrt{-h} h^{\mu \nu}
$$

such that $g$ and $h$ result to be conformally related by

$$
h_{\mu \nu}=\frac{\partial K(\phi, R)}{\partial R} g_{\mu \nu}
$$

We remark again that, once the structural equation is solved, the conformal factor depends just on the values of the matter fields and (the trace of) their stress energy tensors. In other words, the evolution, the definition of the conformal factor and the bi-metric structure is ruled by the values of traces of the stress-energy tensors and by the value of the scalar field $\phi$. In this case, the universality of Einstein field equations does not hold anymore in general. This is evident from (52) where the strong coupling between $R$ and $\phi$ avoids the possibility, also in the vacuum case, to achieve also constant simple solutions for the structural equations (52). We consider furthermore the case when small values of $R$ are considered, corresponding to the very important cases of small curvature spacetimes. As already explained, this limit represent, as a good approximation, the present epoch of the observed universe under suitably regularity conditions. A Taylor expansion of the analytical function $K(\phi, R)$ can be performed:

$$
K(\phi, R)=K_{0}(\phi)+K_{1}(\phi) R+o\left(R^{2}\right)
$$

where only the first leading term in $R$ is considered and we have defined:

$$
\left\{\begin{array}{l}
K_{0}(\phi)=K(\phi, R)_{R=0} \\
K_{1}(\phi)=\left(\frac{\partial K(\phi, R)}{\partial R}\right)_{R=0}
\end{array}\right.
$$

Substituting this expression in (52) and (54) we get (neglecting higher order approximations in $R$ ) the structural equation and the bi-metric structure in this particular case. From the structural equation we get:

$$
R=\frac{1}{K_{1}(\phi)}\left[-\kappa\left(\tau^{\phi}+\tau^{\mathrm{mat}}\right)-2 K_{0}(\phi)\right]
$$

such that the value of the Ricci scalar is always determined, in this first order approximation, in terms of $\left(\tau^{\phi}, \tau^{\text {mat }}, \phi\right)$. The bi-metric structure is otherwise simply defined by means of the first term of the Taylor expansion. We have:

$$
h_{\mu \nu}=K_{1}(\phi) g_{\mu \nu}
$$


which reproduces, as expected, the scalar-tensor case (42). In other words, scalar-tensor theories can be recovered in a first order approximation of a general theory where gravity and non-minimal couplings are any (compare (57) with (46)). This fact agrees with the above considerations where Lagrangians of physical interactions are stochastic functions with local gauge invariance properties [18].

\section{COSMOLOGICAL APPLICATIONS}

The above discussion tells us that, for a given ETG, Palatini approach intrinsically define a bi-metric structure where geodesic and chronological structures of spacetime do not coincide a priori. This fact is extremely relevant in the interpretation of conformal transformations since the interpretation of physical results in the metrics $h_{\mu \nu}$ and $g_{\mu \nu}$ (or alternatively $\bar{g}_{\mu \nu}$ and $g_{\mu \nu}$ )is something different since, in the Palatini formalism $h$ and $g$ are entangled. This means that $g$ provides the chronological structure while $h$ is related to the geodesic structure as the affine connection is assumed to be $\Gamma=\Gamma_{L C}(h)$. This feature assume a crucial role at the level of the solutions which can be worked out in the two dynamics, first of all in cosmology. In fact, a bad interpretation of the geodesic structure of a given spacetime can lead to misunderstand the results and the interpretation of observations. In this section, we want to show how the "same" theory, conformally transformed, can give rise to completely different cosmological solutions. For example, in the Einstein frame we can have solutions with cosmological constant which is the same at every epoch while in the Jordan frame a self-interacting potential and a non-minimal coupling come out. This fact leads to a completely different interpretation of data. The shortcoming is unambiguously solved only if the structure of affine connections is completely controlled as in the Palatini approach.

In order to support these statements, let us take into account scalar-tensor theories in the the Friedmann-Robertson-Walker cosmology. A part the interest of such theories discussed in the Introduction, they are remarkable since, as we have seen, represent the low-curvature limit of general non-minimally coupled higher-order theories whose interpretation is straightforward in the Palatini approach.

Let us assume now that the spacetime manifold is described by a FRW metric. The Lagrangian density (1) takes the form

$$
L_{t}=6 F(\phi) a \dot{a}^{2}+6 F_{\phi}(\phi) a^{2} \dot{a} \dot{\phi}-6 F(\phi) a K+\frac{1}{2} a^{3} \dot{\phi}^{2}-a^{3} V(\phi)
$$

With the subscript $t$, we mean that the time-coordinate considered is the cosmic time $t$ : this 
remark is important for the forthcoming discussion. Here $a$ is the scale factor of the universe and $K$ is spatial curvature constant. The Euler-Lagrange equations relative to (59) are then

$$
\left\{\begin{array}{l}
\frac{2 \ddot{a}}{a}+\frac{\dot{a}^{2}}{a^{2}}+\frac{2 F_{\phi} \dot{a} \dot{\phi}}{F a}+\frac{F_{\phi} \ddot{\phi}}{F}+\frac{K}{a^{2}}+\frac{F_{\phi \phi} \dot{\phi}^{2}}{F}-\frac{\dot{\phi}^{2}}{4 F}+\frac{V}{2 F}=0 \\
\ddot{\phi}+\frac{3 \dot{a} \dot{\phi}}{a}+\frac{6 F_{\phi} \dot{a}^{2}}{a^{2}}+\frac{6 F_{\phi} \ddot{a}}{a}+\frac{6 F_{\phi} K}{a^{2}}+V_{\phi}=0
\end{array}\right.
$$

which correspond to the (generalized) second order Einstein equation and to the Klein-Gordon equation in the FRW case. The energy function relative to (59) is

$$
E_{t}=\frac{\partial L_{t}}{\partial \dot{a}} \dot{a}+\frac{\partial L_{t}}{\partial \dot{\phi}} \dot{\phi}-L_{t}=6 F a \dot{a}^{2}+6 F_{\phi} a^{2} \dot{a} \dot{\phi}+6 F a K+\frac{1}{2} a^{3} \dot{\phi}^{2}+a^{3} V=0
$$

which is the first order generalized Einstein equation.

Performing the conformal transformation defined by (5), (7), (9) on the FRW metric, one should obtain the corresponding expression for the Lagrangian and the corresponding equations of the Einstein-cosmology from the nonstandard coupled Lagrangian (59) and from the generalized Einstein and Klein-Gordon equations, respectively. Unfortunately we see that the presence of the conformal factor (7) implies that the transformed line element which is obtained is no longer expressed in the "cosmic time form". Actually the scale factor of the Einstein theory can be defined as the scale factor of the non-minimally coupled theory multiplied by the conformal factor, but the time coordinate of the Einstein theory has to be redefined if we require to have the cosmic time as well. Absorbing the conformal factor in the redefinition of time, we obtain the transformation on the time coordinate. Therefore, the transformation from the Jordan frame to the Einstein frame in the cosmological case is given by

$$
\left\{\begin{array}{l}
\bar{a}=\sqrt{-2 F(\phi)} a \\
\frac{d \bar{\phi}}{d t}=\sqrt{\frac{3 F_{\phi}^{2}-F}{2 F^{2}}} \frac{d \phi}{d t} \\
d \bar{t}=\sqrt{-2 F(\phi)} d t .
\end{array}\right.
$$

From the Palatini point of view, these transformations are "natural" due to the intrinsic different geodesic structure of the two frames. Furthermore, the system of Eqs. (60), (61) and the relations (62) to pass from the Jordan frame to the Einstein frame are immediately recovered from the Palatini field equations (37) and (39), linked together by the structural equation (46). Moreover in the Palatini formalism, the redefinition of cosmic time in the two frames (i.e. considering $h$ or 
$g$ as the physical metric) naturally follows from (41) and reproduces (62).In other words, Palatini field equations give, at once, dynamics of fields and, being endowed with a bi-metric structure, the relation between the Jordan frame and the Einstein frame.

Using the first and the third of (62), the scale factor $\bar{a}$ in the Einstein frame depends only on $\bar{t}$. The factor $F(\phi)$, which modifies the geodesic structure, is absorbed into the definition of the cosmic time in the Einstein frame. The second of (62) corresponds to the first of relations (9) under the given assumption of homogeneity and isotropy. Under transformation (62) we have that

$$
\begin{aligned}
\frac{1}{\sqrt{-2 F}} L_{t} & =\frac{1}{\sqrt{-2 F}}\left(6 F a \dot{a}^{2}+6 F_{\phi} a^{2} \dot{a} \dot{\phi}-6 F a K+\frac{1}{2} a^{3} \dot{\phi}^{2}-a^{3} V\right)= \\
& =-3 \bar{a} \dot{\bar{a}}^{2}+3 K \bar{a}+\frac{1}{2} \bar{a}^{3} \dot{\bar{\phi}}^{2}-\bar{a}^{3} \bar{V}(\bar{\phi})=\bar{L}_{\bar{t}}
\end{aligned}
$$

in which the dot over barred quantities means the derivative with respect to $\bar{t} ; L_{t}$ is given by (59) and $\bar{L}_{\bar{t}}$ coincides with the "point-like" Lagrangian obtained from the Hilbert-Einstein action plus a scalar field under the assumption of homogeneity and isotropy. In this way, the invariance of the homogeneus and isotropic action under (62) is restored, being $L_{t}$ and $\bar{L}_{\bar{t}}$ equivalent by the (63). The same correspondence as (63) exists between the energy function $E_{t}$ and $\bar{E}_{\bar{t}}$, that is, there is correspondence between the two first order Einstein equations in the two frames. It is interesting to note that the relation (63) reflects the Palatini bi-metric structure: the Lagrangians are equivalent only if the time is conformally transformed and Levi-Civita connection is restored in the new metric.

We focus now our attention on the way in which the Euler-Lagrange equations transform under (62). The Euler-Lagrange equations relative to (63) are the usual second order Einstein equation and Klein-Gordon equation

$$
\left\{\begin{array}{l}
\frac{2 \ddot{\bar{a}}}{\bar{a}}+\frac{\dot{\bar{a}}^{2}}{\bar{a}}+\frac{K}{\bar{a}^{2}}+\frac{1}{2} \dot{\bar{\phi}}^{2}-\bar{V}=0 \\
\ddot{\bar{\phi}}+\frac{3 \dot{\bar{a}} \dot{\bar{\phi}}}{\bar{a}}+\bar{V}_{\bar{\phi}}=0 .
\end{array}\right.
$$

Under (62) it is straightforward to verify that they become

$$
\left\{\begin{array}{l}
\frac{2 \ddot{a}}{a}+\frac{\dot{a}^{2}}{a^{2}}+\frac{2 F_{\phi} \dot{a} \dot{\phi}}{F a}+\frac{F_{\phi} \ddot{\phi}}{F}+\frac{K}{a^{2}}+\frac{F_{\phi \phi} \dot{\phi}^{2}}{F}-\frac{\dot{\phi}^{2}}{4 F}+\frac{V}{2 F}=0 \\
\ddot{\phi}+\frac{3 \dot{a} \dot{\phi}}{a}+\left(\frac{6 F_{\phi} F_{\phi \phi}-F_{\phi}}{3 F_{\phi}^{2}-F}\right) \frac{\dot{\phi}^{2}}{2}+\frac{2 F_{\phi} V}{3 F_{\phi}^{2}-F}-\frac{F V_{\phi}}{3 F_{\phi}^{2}-F}=0
\end{array}\right.
$$


which do not coincide with the Euler-Lagrange equations given by (60). Using the first of (60), the second of (60) can be written as

$$
\begin{aligned}
& \frac{F-3 F_{\phi}^{2}}{F} \ddot{\phi}+\frac{3\left(F-3 F_{\phi}^{2}\right)}{F} \frac{\dot{a} \dot{\phi}}{a}+\left(\frac{F_{\phi}-6 F_{\phi \phi} F_{\phi}}{F}\right) \frac{\dot{\phi}^{2}}{2}+\frac{F_{\phi} \dot{\phi}^{2}}{4 F}-\frac{2 F_{\phi} V}{F}+V_{\phi}+ \\
& +\frac{3 F_{\phi} \dot{a}^{2}}{a^{2}}+\frac{3 F_{\phi} K}{a^{2}}+\frac{3 F_{\phi}^{2} \dot{a} \dot{\phi}}{a}=0,
\end{aligned}
$$

which becomes, taking into account (61)

$$
\frac{F-3 F_{\phi}^{2}}{F} \ddot{\phi}+\frac{3\left(F-3 F_{\phi}^{2}\right)}{F} \frac{\dot{a} \dot{\phi}}{a}+\frac{\dot{\phi}^{2}}{2 F} \frac{d}{d \phi}\left(F-3 F_{\phi}^{2}\right)+\frac{F_{\phi} \dot{\phi}^{2}}{4 F}-\frac{2 F_{\phi} V}{F}+V_{\phi}+\frac{F_{\phi}}{2 a^{3} F} E_{t}=0 .
$$

Comparing (67) with the second of (65), we see that they coincide if $F-3 F_{\phi}^{2} \neq 0$ and $E_{t}=0$. The quantity $F-3 F_{\phi}^{2}$ is proportional to the Hessian determinant of $L_{t}$ with respect to $(\dot{a}, \dot{\phi})$; this Hessian has to be different from zero in order to avoid pathologies in the dynamics [52], while $E_{t}=0$ corresponds to the first order Einstein equation. Clearly, such pathologies are naturally avoided in the Palatini approach where the cosmological equations of motion are derived from the field equations (37) and (39). It is possible to see more clearly at the problem of the cosmological conformal equivalence, formulated in the context of the "point-like" Lagrangian, if we use, as time-coordinate, the conformal time $\eta$, connected to the cosmic time $t$ by the usual relation

$$
a^{2}(\eta) d \eta^{2}=d t^{2}
$$

We can see that the use of $\eta$ makes much easier the treatment of all the problems we have discussed till now. The crucial point is the following: given the form of the FRW line element expressed in conformal time $\eta$ one does not face the problem of redefining time after performing a conformal transformation, since in this case, the expansion parameter appears in front of all the terms of the line element. From this point of view, the conformal transformation which connects Einstein and Jordan frame is given by

$$
\left\{\begin{array}{l}
\bar{a}=\sqrt{-2 F(\phi)} a \\
\frac{d \bar{\phi}}{d \eta}=\sqrt{\frac{3 F_{\phi}^{2}-F}{2 F^{2}}} \frac{d \phi}{d \eta}
\end{array}\right.
$$

where $a, \phi, \bar{a}, \bar{\phi}$ are assumed as functions of $\eta$.

The Hilbert-Einstein "point-like" Lagrangian is given by

$$
\bar{L}_{\eta}=-3 \bar{a}^{\prime 2}+3 K \bar{a}^{2}+\frac{1}{2} \bar{a}^{2} \bar{\phi}^{\prime 2}-\bar{a}^{4} \bar{V}(\bar{\phi})
$$


in which the prime means the derivative with respect to $\eta$, and the subscript $\eta$ means that the time-coordinate considered is the conformal time. Under transformation (69), it becomes

$$
\begin{aligned}
\bar{L}_{\eta} & =-3 \bar{a}^{\prime 2}+3 K \bar{a}^{2}+\frac{1}{2} \bar{a}^{2} \bar{\phi}^{\prime 2}-\bar{a}^{4} \bar{V}(\bar{\phi})= \\
& =6 F(\phi) a^{\prime}+6 F_{\phi}(\phi) a a^{\prime} F^{\prime}(\phi)-6 F(\phi) K a^{2}+\frac{1}{2} a^{2} \phi^{\prime 2}-a^{4} V(\phi)=L_{\eta}
\end{aligned}
$$

which corresponds to the "point-like" Lagrangian obtained from the Lagrangian density in (11) under the hypotheses of homogeneity and isotropy, using the conformal time as time coordinate. This means that the Euler-Lagrange equations relative to (70), which coincides with the second order Einstein equation and the Klein-Gordon equation in conformal time, correspond to the Euler-Lagrange equations relative to (71), under the transformation (69). Moreover, the energy function $\bar{E}_{\eta}$ relative to (70) corresponds to the energy function $E_{\eta}$ relative to (171), so that there is correspondence between the first order Einstein equations. Furthermore, in order to have full coherence between the two formulations, it is easy to verify that, both in the Jordan frame and in the Einstein frame, the Euler-Lagrange equations, written using the conformal time, correspond to the Euler-Lagrange equations written using the cosmic time except for terms in the energy function; for it, one gets the relation

$$
E_{\eta}=a E_{t}
$$

which holds in both the frames; thus the first order Einstein equation is preserved under the transformation from $\eta$ to $t$ and there is full equivalence between the two formulations. We want to point out that for the two Lagrangians $L_{\eta}$ and $L_{t}$ the same relation as (72) holds. On the other hand, such results naturally hold if one takes into account the relation (42) derived from the second Palatini equation (37).

When ordinary matter is present the standard Einstein (cosmological) "point-like" Lagrangian is

$$
\bar{L}_{t o t}=\bar{L}_{\bar{t}}+\bar{L}_{m a t}
$$

in which $\bar{L}_{\bar{t}}$ is given by (63) and $\bar{L}_{\text {mat }}$ is the Lagrangian relative to perfect fluid matter. Using the contracted Bianchi identity, it can be seen that $\bar{L}_{\text {mat }}$ can be written as 53 ]

$$
\bar{L}_{m a t}=-D \bar{a}^{3(1-\gamma)}
$$

where $D$ is connected to the total amount of matter. In writing (73) and (174) we have chosen the cosmic time as time-coordinate. Under the transformation (62) we have, besides relation (63), 
that (74) corresponds to

$$
\bar{L}_{\text {mat }}=(\sqrt{-2 F})^{3(1-\gamma)} L_{m a t},
$$

where, analogously to (74)

$$
L_{m a t}=D a^{3(1-\gamma)}
$$

Then we have that, using (62), (73), it becomes

$$
\frac{1}{\sqrt{-2 F}} L_{t o t}=\frac{1}{\sqrt{-2 F}}\left[L_{t}+(\sqrt{-2 F})^{(4-3 \gamma)} L_{m a t}\right]
$$

in which we have defined the total "point-like" Lagrangian after the conformal transformation as

$$
L_{t o t}=L_{t}+(\sqrt{-2 F})^{(4-3 \gamma)} L_{m a t}
$$

(cfr. (63) ); the transformation of $\bar{L}_{\text {tot }}$ under (62) has to be written following the expression (77) and consequently the "point-like" Lagrangian $L_{t o t}$ has to be defined as in (78).

Summarizing, the perfect fluid-matter, which minimally interact in the Jordan frame, results

non-minimaly interacting in the conformally transformed Einstein frame unless $\gamma=\frac{4}{3}$ (radiation), since the standard matter Lagrangian term is coupled with the scalar field in a way which depends on the coupling F. Such a coupling between the matter and the scalar field is an effect of the transformation, therefore depending on the coupling. Also this interaction which emerges passing from the Jordan frame to the Einstein frame, is immediately recovered considering the Palatini structural equation (46) and follows directly from (47) which express the relation between the different metrics (and consequently between the two frames).

\section{A. Some relevant examples}

The exact identification of the frame is crucial when the solutions are matched with data. We are going to give some examples where the nature of solutions drastically changes considering the Einstein frame or the Jordan frame without taking into account the problem of transformations of physical quantities between them. The ambiguity is removed in the Palatini approach since, due to the intrinsic bi-metric structure, the two frames are given together by the same dynamics.

i) Let us consider a model in the Einstein frame with a scalar field, a constant potential and zero curvature. The Lagrangian is given by

$$
\bar{L}_{\bar{t}}=-3 \bar{a} \dot{\bar{a}}^{2}+\frac{1}{2} \bar{a}^{3} \dot{\bar{\phi}}^{2}-\bar{a}^{3} \Lambda
$$


the Euler-Lagrange equations and the energy condition are

$$
\left\{\begin{array}{l}
\frac{2 \ddot{\bar{a}}}{\bar{a}}+\frac{\dot{\bar{a}}^{2}}{\bar{a}^{2}}+\frac{1}{2} \dot{\bar{\phi}}^{2}-\Lambda=0 \\
\ddot{\bar{\phi}}+\frac{3 \dot{\bar{a}} \dot{\bar{\phi}}}{\bar{a}}=0 . \\
\frac{\dot{\bar{a}}^{2}}{\bar{a}^{2}}-\frac{1}{3}\left(\frac{1}{2} \dot{\bar{\phi}}^{2}+\Lambda\right)=0 .
\end{array}\right.
$$

The system can be easily solved giving the solution

$$
\left\{\begin{array}{l}
\bar{a}=\left[c_{1} e^{\sqrt{3 \Lambda} \bar{t}}-\frac{\dot{\bar{\phi}}_{0}^{2}}{8 \Lambda c_{1}^{2}} e^{-\sqrt{3 \Lambda} \bar{t}}\right]^{\frac{1}{3}} \\
\bar{\phi}=\bar{\phi}_{0}+\sqrt{\frac{2}{3}} \ln \frac{1-\frac{\dot{\bar{\phi}}_{0}}{2 c_{1} \sqrt{2 \Lambda}} e^{-\sqrt{3 \Lambda} \bar{t}}}{1+\frac{\dot{\bar{\phi}}_{0}}{2 c_{1} \sqrt{2 \Lambda}} e^{-\sqrt{3 \Lambda} \bar{t}}}
\end{array}\right.
$$

Three integration constants appear in the solution, since Eq. 818 corresponds to a constraint on the value of the first integral $\bar{E}_{\bar{t}}$. We have that, in the limit of $\bar{t} \rightarrow+\infty$, the behavior of $\bar{a}$ is exponential with characteristic time given by $\sqrt{\frac{\Lambda}{3}}$, as we would expect, and $\bar{\phi}$ goes to a constant. Looking at the second of (9), we have that such a model in the Einstein frame corresponds, in the Jordan frame, to the class of models with (arbitrarily given) coupling $F$ and potential $V$ connected by the relation

$$
\frac{V}{4 F^{2}}=\Lambda
$$

the solution of which can be obtained from (82) via the transformation (62). We can thus fix the potential $V$ and obtain, from (83), the corresponding coupling. This can be used as a method to find the solutions of non-minimally coupled models with given potentials, the coupling being determined by (83). In other words, a single model in the Einstein frame corresponds to a family of models in the Jordan frame, but giving "a priori" the bi-metric structure of the theory by the Palatini approach, the model is only one. As an example, let us take into account the case

$$
V=\lambda \phi^{4}, \quad \lambda>0
$$

which correspond to a "chaotic inflationary" potential [59]. The corresponding coupling is quadratic in $\phi$

$$
F=k_{0} \phi^{2}
$$


in which

$$
k_{0}=-\frac{1}{2} \sqrt{\frac{\lambda}{\Lambda}}
$$

Substituting (82) into (62), we get

$$
\left\{\begin{array}{l}
a=\frac{\bar{a}}{\phi \sqrt{-2 k_{0}}} \\
d \phi=\phi \sqrt{\frac{2 k_{0}}{12 k_{0}-1}} d \bar{\phi} \\
d t=\frac{d \bar{t}}{\phi \sqrt{-2 k_{0}}} .
\end{array}\right.
$$

As we see from these relations, it has to be $k_{0}<0$. Integrating the second of (67), we have the conformal relation between the scalar fields, i.e. $\phi$ in terms of $\bar{\phi}$

$$
\phi=\alpha_{0} e^{\sqrt{\frac{2 k_{0}}{12 k_{0}-1}} \bar{\phi}} .
$$

Substituting (88) in the first of (87) and taking into account the second of (82), we have the solutions $a$ and $\phi$ as functions of $\bar{t}$

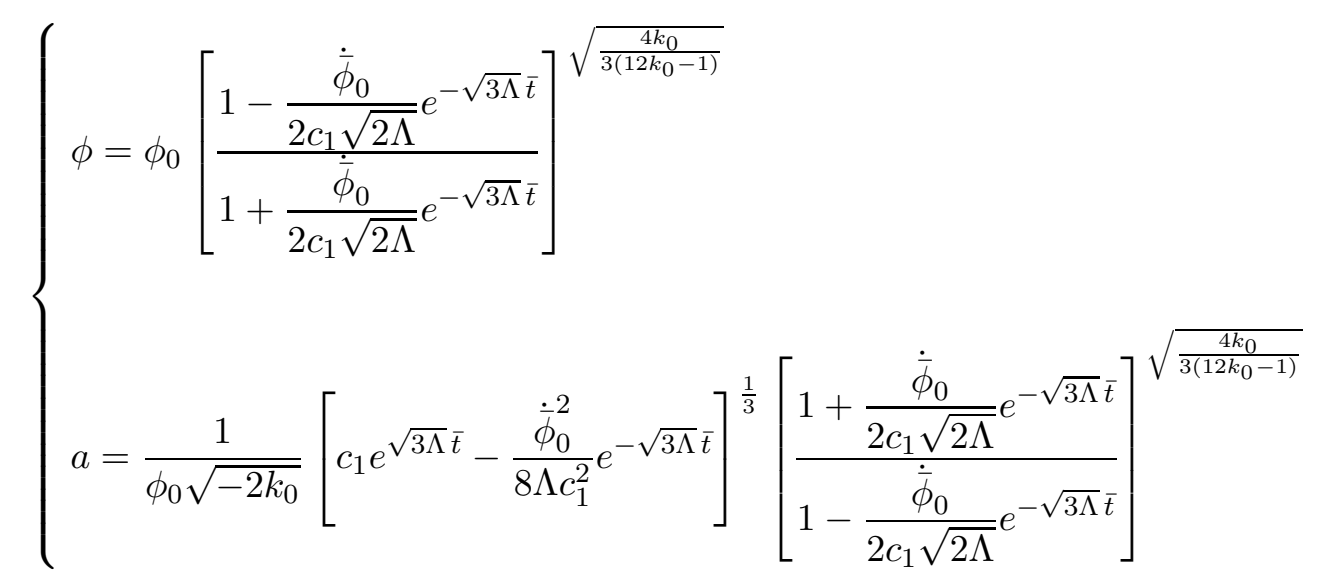

in which $\phi_{0}=\alpha_{0} e^{\sqrt{\frac{2 k_{0}}{12 k_{0}-1}} \bar{\phi}_{0}}$. Substituting (88) in the third of (87), taking into account (82), we get

$$
d t=\frac{d \bar{t}}{\phi_{0} \sqrt{-2 k_{0}}}\left[\frac{1+\frac{\dot{\bar{\phi}}_{0}}{2 c_{1} \sqrt{2 \Lambda}} e^{-\sqrt{3 \Lambda} \bar{t}}}{1-\frac{\dot{\bar{\phi}}_{0}}{2 c_{1} \sqrt{2 \Lambda}} e^{-\sqrt{3 \Lambda} \bar{t}}}\right]^{\sqrt{\frac{4 k_{0}}{3\left(12 k_{0}-1\right)}}} .
$$

We obtain $\bar{t}$ as a function of $t$ integrating (90) and then considering the inverse function; Eq.(90) could be easily integrated if the exponent $\sqrt{\frac{4 k_{0}}{3\left(12 k_{0}-1\right)}}$ would be equal to \pm 1 , but this corresponds 
to a value of $k_{0}=\frac{3}{32}$ which is positive and thus it turns out to be not physically acceptable. In general, (90) is not of easy solution. We can analyze its asymptotic behavior, obtaining

$$
\frac{d t}{d \bar{t}} \stackrel{\bar{t} \rightarrow+\infty}{\rightarrow} \frac{1}{\phi_{0} \sqrt{-2 k_{0}}}
$$

that is, asymptotically,

$$
t-t_{0} \simeq \frac{\bar{t}}{\phi_{0} \sqrt{-2 k_{0}}}
$$

Substituting (92) in the asymptotic expression of (89), we obtain the asymptotic behavior of the solutions (since from (91) one has $t^{\bar{t} \rightarrow+\infty}+\infty$ )

$$
\left\{\begin{array}{l}
a \simeq \frac{c_{1}^{1 / 3}}{\phi_{0} \sqrt{-2 k_{0}}} e^{\phi_{0} \sqrt{\frac{-2 \Lambda k_{0}}{3}}\left(t-t_{0}\right)} \\
\phi \simeq \phi_{0} .
\end{array}\right.
$$

Thus we have that, asymptotically, $a(t)$ is exponential, and $\phi(t)$ is constant; the coupling $F$ is asymptotically constant too, so that, fixing the arbitrary constant of integration to obtain the finite transformation of $\bar{a}, \bar{\phi}$ (that is, fixing the units, see [35]), once $k_{0}$ is fixed, it is possible to recover asymptotically the Einstein gravity from the Jordan frame.

As a remark we would like to notice that the asymptotic expression (93) of $a(t)$ and $\phi(t)$ are solutions of the Einstein equations and Klein-Gordon equation with zero curvature and $F$ and $V$ given by (84), (85) . They have not been obtained as solutions of the asymptotic limits of these equations. It means then that they are, in any case, particular solutions of the given non-minimally coupled model.

ii) Another interesting case is the Ginzburg-Landau potential

$$
V=\lambda\left(\phi^{2}-\mu^{2}\right)^{2}, \quad \lambda>0
$$

The corresponding coupling is given by

$$
F=k_{0}\left(\phi^{2}-\mu^{2}\right)
$$

in which $k_{0}$ is given by (86) when $\phi^{2}>\mu^{2}$ while is given by (86) with opposite sign when $\phi^{2}<\mu^{2}$, in order to have $F<0$. With this coupling, the corresponding conformal transformation turns out to be singular for $\phi^{2}=\mu^{2}$, thus with this method it is not possible to solve this model for $\phi$ equal to the Ginzburg-Landau mass $\mu$. The explicit function $\phi=\phi(\bar{\phi})$ is obtained inverting the integral

$$
\bar{\phi}-\bar{\phi}_{0}=\int \frac{\left[3 \sqrt{\frac{\lambda}{\Lambda}} \phi^{2}+\frac{1}{2}\left(\phi^{2}-\mu^{2}\right)\right]^{\frac{1}{2}}}{\left[\frac{\lambda}{4 \Lambda}\right]^{\frac{1}{4}}\left(\phi^{2}-\mu^{2}\right)} d \phi ;
$$


and it is possible to carry analogous considerations as in the previous case, concluding that asymptotically the behavior of $a(t)$ is exponential and that of $\phi(t)$ is constant.

iii) Another interesting case is

$$
V=\lambda \phi^{2}, \quad \lambda>0 ; \quad F=k_{0} \phi^{2}, \quad k_{0}<0
$$

in the Jordan frame. The coupling is the same as in (85D) and the conformal transformation is given by (87). To obtain the corresponding potential in the Einstein frame we have to substitute (88) in the relation

$$
\bar{V}(\bar{\phi})=\frac{\lambda}{4 k_{0}^{2} \phi^{2}(\bar{\phi})}
$$

that is

$$
\bar{V}(\bar{\phi})=\frac{\lambda}{4 k_{0}^{2} \phi_{0}^{2}} e^{-2 \sqrt{\frac{2 k_{0}}{12 k_{0}-1}} \bar{\phi}},
$$

which gives, in the Einstein frame, power-law solutions [61, 62]. A general remark concerns the relation between the Hubble parameter in the Einstein and in the Jordan frame. It is

$$
\bar{H}=\frac{\dot{\bar{a}}}{\bar{a}}=\frac{1}{(-2 F)}\left(-\frac{\dot{F}}{\sqrt{-2 F}}+\sqrt{-2 F} \frac{\dot{a}}{a}\right)=\frac{\dot{F}}{2 F \sqrt{-2 F}}+\frac{H}{\sqrt{-2 F}},
$$

in which we have used the relations (62). Relation (100) is useful to study the asymptotic behavior of the Hubble parameter: if we require an asymptotic de Sitter-behavior in both the Einstein and Jordan frame (for example, in order to reproduce quintessential accelerated behavior), we have to require $\bar{H} \stackrel{\bar{t} \rightarrow+\infty}{\rightarrow} \bar{C}$ and $H \stackrel{t \rightarrow+\infty}{\rightarrow} C$ where $\bar{C}$ and $C$ are constants, from (100), we obtain a differential equation for the coupling $F$ as a function of $t(t>>0)$, given by

$$
\dot{F}+2 C F-2 \bar{C} F \sqrt{-2 F}=0 .
$$

Its solution is

$$
F=-\frac{C^{2}}{2 \bar{C}^{2}}\left[\frac{1}{1-F_{0} e^{C t}}+1\right]^{2},
$$

in which $F_{0}$ is the integration constant; this is the time-behavior that $F$ has to assume on the solution $\phi(t)$, in order to have a de Sitter asymptotical accelerated behavior in both frames. It easy to verify that both the couplings in the examples i) and ii) satisfy (102) asymptotically. 


\section{CONCLUSIONS}

The decennial debate about the physical relevance of conformal transformations can be enlightened by taking into account the Palatini approach to the Extended Theories of gravity. In this framework, the conformal transformation is not only a mathematical tool capable of disentangling matter from gravitational degrees of freedom, but it is related to the bi-metric structure of spacetime where chronological structure and geodesic structure are, a priori, independent. While in Hilbert-Einstein gravity the affine connections can be assumed in any case Levi-Civita, this is not true in the Palatini approach, being the fields $g$ and $\Gamma$ independent. Due to this fact, the ambiguities to work out a given theory in the Einstein or in the Jordan frame are removed since the Palatini field equations and, first of all the structural equation of spacetime, give, at the same time, information on both frames. In other words, discussing if "Jordan" or "Einstein" is the true physical frame is a nonsense in the Palatini approach.

In this paper, we have taken into account scalar-tensor, higher-order and higher-order-scalartensor theories of gravity showing how the Palatini field equations furnish the conformal structure. Furthermore, we have shown that higher-order and scalar-tensor theories can be dealt under the same standard: in particular, scalar-tensor (second-order) theories can be recovered in the limit of small curvatures.

These results become crucial in cosmology since, by them, it is possible to show that solutions taken into account as different ones are the same in the Palatini approach. For example, the recently observed acceleration of the Hubble fluid [63, 64, 65, 66, 67] is an evidence that some form of "dark energy" should be present in the cosmic dynamics. Despite of this general result, such an accelerated dynamics can be achieved in several ways (cosmological constant [51], scalar fields dynamics [68], curvature quintessence [69]) but no definite answer, up to now, has been given about its nature. In what we have discussed, we have shown that a cosmic dynamics ruled by the cosmological constant in the Einstein frame becomes ruled by a non-minimally coupled, selfinteracting scalar field (evolving in time) in the Jordan frame. Consequently, matching the data against a solution in the Einstein frame or in the Jordan frame could lead to highly misleading results and interpretation. The shortcoming is completely overcome in the Palatini approach which furnishes, at the same time, dynamics and conformal structure of the given ETG avoiding such ambiguities. These considerations have to be further developed considering concretely the matching with the data.

\section{Acknowledgements}


We are grateful for useful discussions and suggestions to V. Cardone, S. Carloni and A. Troisi. This work is partially supported (G.A. and M.F.) by GNFM-INdAM research project "Metodi geometrici in meccanica classica, teoria dei campi e termodinamica" and by MIUR: PRIN 2003 on "Conservation laws and thermodynamics in continuum mechanics and field theories". G.A. is supported by the I.N.d.A.M. grant: "Assegno di collaborazione ad attivitá di ricerca a.a. 20022003".

[1] A. Guth, Phys. Rev. D 23, 347 (1981).

[2] S. Weinberg, Gravitation and Cosmology, Wiley, 1972 New York N.Y.

[3] C. Brans and R.H. Dicke, Phys. Rev. 124, 925 (1961).

[4] A.A. Starobinsky, Phys. Lett. 91B (1980) 99.

[5] D. La and P.J. Steinhardt, Phys. Rev. Lett. 62 (1989) 376.

[6] A. Vilenkin, Phys. Rev. 32 D (1985) 2511. E. Carugno, S. Capozziello, F. Occhionero, Phys. Rev. D 47(1993) 4261.

[7] I.L. Buchbinder, S.D. Odintsov, and I.L. Shapiro, Effective Action in Quantum Gravity, IOP Publishing (1992) Bristol.

[8] C.M. Will, Theory and Experiments in Gravitational Physics (1993) Cambridge Univ. Press, Cambridge.

[9] K. Stelle, Gen. Relativ. Grav. 9 (1978) 353.

[10] R.H. Sanders, Ann. Rev. Astr. Ap. 2 (1990) 1.

[11] P.D. Mannheim and D. Kazanas, Ap. J. 342 (1989) 635. O.V. Barabash and Yu. V. Shtanov, Phys. Rev. 60 D (1999) 064008.

[12] J.D. Anderson et al., Phys. Rev. Lett. 81 (1998) 2858.

J.D. Anderson, et al., Phys. Rev. D 65 (2002) 082004.

[13] I. Quant and H.-J. Schmidt, Astron. Nachr. 312 (1991) 97.

[14] P. Schneider, J. Ehlers, and E.E. Falco, Gravitational Lenses Springer-Verlag (1992) Berlin.

[15] L.M. Krauss and M. White, Ap. J. 397 (1992) 357.

[16] L. Nottale in Dark Matter (Moriond Astrophysics Meetings), J. Andouze and J. Tran Thanh Van eds. (1988) Frontieres, Gif-sur-Yvette.

[17] G. Magnano, M. Ferraris, and M. Francaviglia, Gen. Relativ. Grav. 19, 465 (1987).

Ferraris M, Francaviglia M and Magnano G 1990 Class. Quantum Grav. 7261

[18] J. Barrow and A.C. Ottewill, J. Phys. A: Math. Gen. 16, 2757 (1983).

[19] N.D. Birrell and P.C.W. Davies, Quantum Fields in Curved Space, Cambridge Univ. Press, Cambridge (1982).

[20] G. Vilkovisky, Class. Quantum Grav. 9, 895 (1992).

[21] M. Gasperini and G. Veneziano, Phys. Lett. 277B (1992) 256. 
[22] H. Bondi, Cosmology, Cambridge Univ. Press, Cambridge (1952).

[23] S. Capozziello, R. de Ritis, C. Rubano, and P. Scudellaro, La Rivista del Nuovo Cimento 4 (1996) 1.

[24] D.W. Sciama, Mon. Not. R. Ast. Soc. 113, 34 (1953).

[25] P. Teyssandier and Ph. Tourrenc, J. Math. Phys. 24, 2793 (1983).

[26] K. Maeda, Phys. Rev. D 39, 3159 (1989).

[27] D. Wands, Class. Quantum Grav. 11, 269 (1994).

S. Capozziello, R. de Ritis, A.A. Marino. Gen. Relativ. Grav. 30, 1247, (1998).

S. Capozziello, G. Lambiase, Gen. Relativ. Grav. 32, 295 (2000).

[28] S. Gottlöber, H.-J. Schmidt, and A.A. Starobinsky, Class. Quantum Grav. 7, 893 (1990).

[29] T.V. Ruzmaikina and A.A. Ruzmaikin, JETP, 30, 372 (1970).

[30] L. Amendola, A. Battaglia-Mayer, S. Capozziello, S. Gottlöber, V. Müller, F. Occhionero and H.-J. Schmidt, Class. Quantum Grav. 10, L43 (1993).

[31] A. Battaglia-Mayer and H.-J. Schmidt, Class. Quantum Grav. 10, 2441 (1993).

[32] H.-J. Schmidt, Class. Quantum Grav. 7, 1023 (1990).

[33] L. Amendola, S. Capozziello, M. Litterio, F. Occhionero, Phys. Rev. D 45, 417 (1992).

[34] Higgs P W 1959 Nuovo Cim. 11816

Whitt B 1984 Phys. Lett. 145B 176

Ferraris M, Francaviglia M and Magnano G 1988 Class. Quantum Grav. 5 L95

Jakubiec A and Kijowski J 1988 Phys. Rev. B 371406.

[35] Sokolowski L M 1989 Class. Quantum Grav. 62045

[36] G. Magnano and L.M. Sokołowski, Phys. Rev. D50, 5039 (1994), (gr-qc/9312008); G. Magnano, Proceedings of XI Italian Conference on General Relativity and Gravitation, Trieste, (1994), (gr-qc/9511027).

[37] S. Capozziello, R.de Ritis, C. Rubano, P. Scudellaro, Int. Journ. Mod. Phys. D 5, 85 (1996).

[38] Dicke R H 1962 Phys. Rev. 1252163

[39] Bekenstein J D 1974 Ann. Phys. (N.Y.) 82535

Singh T and Singh Tarkeshwar 1987 Int. Journ. Mod. Phys. A 2645

Schmidt H J 1988 Phys. Lett. 214B 519

Madsen M S 1988 Class. Quantum Grav. 5627

Damour T and Esposito-Farèse G 1992 Class. Quantum Grav. 92093

S. Capozziello, M. Demianski, R. de Ritis, and C. Rubano, Phys. Rev. D 52, 3288 (1995).

[40] V. Faraoni, Cosmology in Scalar-Tensor Gravity, Kluwer Academic, Dordrecht (2004).

[41] Torres D F and Vucetich H 1996 Phys. Rev. D 547373

[42] Synge J L 1960 Relativity. The general theory (Amsterdam: North Holland)

Parker L 1973 Phys. Rev. D 7976

Wald R M 1984 General Relativity (Chicago: University of Chicago Press)

[43] Damour T, Gibbons G W and Gundlach C 1990 Phys. Rev. Lett. 64123 
[44] Jordan P 1955 Schwerkraft und Weltall (Braunschweig: Vieweg); 1959157112

Fierz M 1956 Helv. Phys. Acta 29128

[45] A. Einstein, Sitzung-ber. Preuss. Akad. Wiss., 414 (1925).

[46] M. Ferraris, M. Francaviglia, C. Reina, Gen. Relativ. Grav. 14, 243 (1982).

[47] H.A. Buchdahl, J. Phys. A 12 (8) (1979), 1229;

H.A. Buchdahl, Proc. Camb. Phil. Soc. 68 (1960), 396.

[48] M. Ferraris, M. Francaviglia and I. Volovich, Nuovo Cim. B108, 1313 (1993) (gr-qc/9303007); M. Ferraris, M. Francaviglia and I. Volovich, Class. Quant. Grav. 11, 1505 (1994).

[49] G. Allemandi, A. Borowiec and M. Francaviglia, Phys.Rev. D - to appear (hep-th/0403264).

[50] D.N. Vollick, Phys. Rev. D68, 063510 (2003) (astro-ph/0306630); X.H. Meng and P. Wang, Class. Quant. Grav. 20, 4949-4962 (2003) (astro-ph/0307354); X.H. Meng and P. Wang, Class. Quant. Grav. 21, 951-960 (2004) (astro-ph/0308031); X.H. Meng and P. Wang, (astro-ph/0308284); X.H. Meng and P. Wang, (hep-th/0309062); G.M. Kremer and D.S.M. Alves, (gr-qc/0404082).

[51] V. Sahni and A. Starobinsky, Int. J. Mod. Phys. D9, 373-444 (2000) (astro-ph/9904398)

[52] S. Capozziello and R. de Ritis, Phys. Lett. 177 A (1993) 1.

S. Capozziello and R. de Ritis, Class. Quantum Grav. 11 (1994) 107.

[53] Capozziello S and de Ritis R 1994 Phys. Lett. 195A 48

[54] M.B. Mijic, M.S. Morris and W. Suen, Phys. Rev. 34D, 2934 (1986).

[55] Bergmann P G 1968 Int. Journ. Theor. Phys. 125

Piccinelli G, Lucchin F and Matarrese S 1992 Phys. Lett. 277B 58

Mollerach S and Matarrese S 1992 Phys. Rev. D 451961

Barrow J D and Maeda K 1990 Nucl. Phys. B 341294

Barrow J D 1993 Phys. Rev. D 475329

Cotsakis S and Flessas G 1993 Phys. Rev. D 483577

[56] Salopek D, Bond J and Bardeen J 1989 D 401753

Gibbons G W and Maeda K 1988 Nucl. Phys. B 298741

Kolb E W, Salopek D and Turner M 1990 Phys. Rev. D 4123925

Deruelle N, Garriga J and Verdaguer E 1991 Phys. Rev. D 431032

[57] Demianski M, de Ritis R, Rubano C and Scudellaro P 1992 Phys. Rev. D 461391

Madsen M S, Mimoso P J, Butcher J A and Ellis G F R 1992 Phys. Rev. D 461399

[58] Capozziello S and de Ritis R 1996 astro-ph 9605070

Capozziello S, de Ritis R and Marino A A 1996 Helv. Phys. Acta 69241

[59] Linde A D 1982 Phys. Lett. 108B 389

Olive K A 1990 Phys. Rep. 190307

[60] Gradshteyn I S and Ryzhik I M 1980 Table of Integrals, Series, and Products (New York: Academic)

[61] Lucchin F and Matarrese S 1985 Phys. Rev. D 321316

[62] Burd A B and Barrow J D 1988 Nucl. Phys. B 308929 
[63] J.L. Tonry, B.P. Schmidt, B. Barris et al. Ap. J. 594, 1 (2003).

[64] P. de Bernardis, et al., Nature 404, 955 (2000).

[65] S. Perlmutter et al., Ap. J. 517, 565 (1999) (astro-ph/9812133); S. Perlmutter el al., Nature 404, 955 (2000).

[66] A.G. Riess et al., Ap. J., 116, 1009 (1998) (astro-ph/9805201).

[67] L. Verde et al., MNRAS, 335, 432 (2002).

[68] P.J. Steinhardt, L.Wang, I. Zlatev, Phys. Rev. D 59, 123504 (1999); B. Boisseau, G. Esposito-Farese, D. Polarski, A.A. Starobinsky, Phys. Rev. Lett. 85, 2236 (2000); P.Ferreira and M.Joyce, Phys. Rev. D57, 6022 (1998).

[69] S.M. Carroll, V. Duvvuri, M. Trodden and M.S. Turner, (astro-ph/0306438); S. Capozziello, S. Carloni and A. Troisi, "Recent Research Developments in Astronomy and Astrophysics" -RSP/AA/21-2003 (astro-ph/0303041); S. Capozziello, Int. J. Mod. Phys. D11, 483 (2002); S. Capozziello, V.F. Cardone, S. Carloni and A. Troisi, Int. J. Mod. Phys. D12, 1969 (2003). S. Nojiri and S.D. Odintsov, Phys. Rev. D68, 123512 (2003) (hep-th/0307288); S. Nojiri and S.D. Odintsov, to appear in GRG (hep-th/0308176); T. Chiba, Phys. Lett. B575, 1-3 (2003) (astro-ph/0307338); D. Barraco, V.H. Hamity and H. Vucetich, Gen. Rel. Grav. 34 (4), 533-547 (2002); R. Dick, (gr-qc/0307052). E.E. Flanagan, Phys. Rev. Lett. 92, 071101 (2004) (astro-ph/0308111); E.E. Flanagan, Class. Quant. Grav. 21, 417-426 (2003) (gr-qc/0309015); D.N. Vollick, (gr-qc/0312041); E.E. Flanagan, (gr-qc/0403063). G.J. Olmo and W. Komp, (gr-qc/0403092); A.D. Dolgov and M. Kawasaki, Phys. Lett. B573, 1-4 (2003) (astro-ph/0307285); P. Wang, G.M. Kremer, D.S.M. Alves and X-H. Meng, (gr-qc/0408058). 